\title{
Localization Transition in Incommensurate non-Hermitian Systems
}

\author{
Amin Jazaeri and Indubala I. Satijal \\ Department of Physics and \\ School of Computational Sciences \\ George Mason University, \\ Fairfax, VA 22030
}

(November 15, 2018)

A class of one-dimensional lattice models with incommensurate complex potential $V(\theta)=2\left[\lambda_{r} \cos (\theta)+i \lambda_{i} \sin (\theta)\right]$ is found to exhibit localization transition at $\left|\lambda_{r}\right|+\left|\lambda_{i}\right|=1$. This transition from extended to localized states manifests in the behavior of the complex eigenspectum. In the extended phase, states with real eigenenergies have finite measure and this measure goes to zero in the localized phase. Furthermore, all extended states exhibit real spectrum provided $\left|\lambda_{r}\right| \geq\left|\lambda_{i}\right|$. Another novel feature of the system is the fact that the imaginary part of the spectrum is sensitive to the boundary conditions only at the onset to localization.

75.30.Kz, 64.60.Ak, 64.60.Fr

Incommensurate systems such as the Harper equation [2] provide an important class of models exhibiting both extended and localized states in one dimension (1D). In this paper, we study the localization transition in systems with competing length scales where the underlying potential is complex. The systems under investigation are described by the following class of lattice tight binding models (tbm),

$$
\psi_{n+1}+\psi_{n-1}+2\left[\lambda_{r} \cos \left(\theta_{n}\right)+i \lambda_{i} \sin \left(\theta_{n}\right)\right] \psi_{n}=E \psi_{n},
$$

where $\theta_{n}=2 \pi \sigma n+\alpha$. Here, $\alpha$ is a constant phase factor and $\sigma$ is an irrational number, which we choose to be the the golden mean. This lattice model describes a system where the period of the potential is incommensurate with the periodicity of the lattice. For $\lambda_{i}=0$, it reduces to the Harper equation which exhibits a localization transition at $\left|\lambda_{r}\right|=1$. Recently, non-Hermitian systems have been the subject of various theoretical [3 [6] and experimental [7,8] studies. In certain 1D random systems, where all states for the Hermitian problem are localized, the addition of a complex vector potential has been shown [4] to result in the delocalization of states, accompanied by the eigenvalues becoming complex. The system we investigate exhibits both extended and localized states and hence facilitates the study of non-Hermiticity on both these phases. By investigating how the nonHermiticity alters the localization transition and as well as the eigenenergies in the complex plane, we are able to study the correlation between the nature of the eigenspectrum and the transport characteristics of the model. It is implicit in the literature [9] that non-Hermiticity corresponds to dissipation and decoherence and as such we arguably explore the impact of these effects on the localization transition. Two interesting limits that we explore in detail are the case of pure imaginary potential $\lambda_{r}=0$ and the case where the real and the imaginary parts are equal $\left(\lambda_{r}=\lambda_{i}\right)$ which can be described as the strong and the weak dissipative limits, respectively. Further, we also study the case of non-Hermitian lattice models with real spectra. This is an interesting problem, since certain complex potentials in quantum mechanics are known to have a real spectrum provided the potentials exhibit parity and time reversal(PT) symmetry [3]. Here, we seek the criterion for a real spectrum in non-Hermitian lattice models exhibiting localization transition.

We study periodic boundary conditions (PBC) antiperiodic boundary conditions (APBC) and free boundary conditions to investigate the sensitivity to the boundary effects. As expected, only extended states are sensitive to the boundary conditions and this sensitivity to the boundary effects can be used to distinguish extended and localized states. We use $\Delta E_{r}$, which is the real part of difference in the eigenenergies between $\mathrm{PBC}$ and $\mathrm{APBC}$, summed over all states, to distinguish extended and localized states: The extended states are characterized by finite value of $\Delta E_{r}$ while in the localized phase $\Delta E_{r}=0$, reflecting its insensitivity to changes at the boundaries. Our detailed numerical study, based on sensitivity to various boundary conditions and wave functions shows that the non-Hermitian system exhibits localization transition at $\left|\lambda_{r}\right|+\left|\lambda_{i}\right|=1$. This implies that the Hermitian and the non-Hermitian part of the potential carry the same weight in determining the transport characteristics of the model. Fig. (1) shows $\Delta E_{r}$ and $\Delta E_{i}$ for weak dissipation limit $\lambda_{r}=\lambda_{i}=\lambda$, which is discussed in detail below. A rather intriguing result is that the imaginary part of the $\Delta E$ is sensitive to the boundary effects only at the onset to localization transition. This result found to be true for other parameter values implies that the life time of the metastable system depends upon the boundary conditions only at the transition point.

In order to explore the relationship between the localization character and the behavior of the eigenenergies, we have extensively studied of the eigenspectrum in the two-parameter $\left(\lambda_{r}, \lambda_{i}\right)$ space. Figs. (2-4) show the variation in eigenenergies with the parameters for some special cases. These studies suggest that it is the localized phase of the non-Hermitian lattice model (1) that is 
characterized by a complex spectrum. in contrast to previous results [4] where the spectra becomes complex when the localized states become delocalized. In the extended phase, the spectrum is real provided $\left|\lambda_{r}\right| \geq \mid \lambda_{i}$. Furthermore, for $\left|\lambda_{r}\right|<\left|\lambda_{i}\right|$, the number of extended states with real eigenenergies have finite measure. Therefore, the fraction of states with real spectrum is finite in extended phase and vanishes in localized phase. This provides a new order parameter for the localization transition, as shown in Fig. (5).

In contrast with the earlier results [4] where a nonHermitian vector potential was found to delocalize the localized states of the random system, the addition of a non-Hermitian potential to the Harper model which exhibits both extended and localized states does not alter the localization character of the system. Furthermore, our results associate a complex spectrum with the localized states, in marked contrast with the earlier result where complex spectrum implied delocalization. This is one of the central result of our analysis. It implies that the previous results relating complex eigenvalues and delocalization must be understood as special to the type of the system investigated, namely random system with non-Hermitian vector potential, and may not describe the generic property of non-Hermitian systems exhibiting localization.

We now discuss the two limiting cases: the strong and the weak dissipation limit. The first case corresponds to pure imaginary potential $\left(\lambda_{r}=0\right)$. It is interesting to note that the model with pure imaginary potential exhibits duality very similar to that of the pure Hermitian problem [10]. Under the Fourier transformation (FT),

$$
\psi_{n}=\sum e^{i \theta_{n} m} \phi_{m}
$$

the tbm (1) with $\lambda \equiv \lambda_{i}$ reduces to

$$
\phi_{m+1}+\phi_{m-1}+\frac{2 i}{(\lambda)} \cos \left(\theta_{m}\right) \phi_{m}=-\frac{i E}{\lambda} \phi_{m} .
$$

Comparing this with the original model, we obtain that

$$
E^{*}(\lambda)=-\frac{i}{\lambda} E\left(\frac{1}{\lambda}\right)
$$

This implies that the real and the imaginary part of the eigenenergies are related as $E_{r}(\lambda)=\frac{1}{\lambda} E_{i}\left(\frac{1}{\lambda}\right)$. Therefore the case of the pure imaginary potential has the interesting property that the localization transition interchanges the real and the imaginary part of the spectrum. At the onset to localization $(\lambda=1)$ the model is self-dual, with $E_{r}=E_{i}$. Fig. (6) shows eigenenergies at some values of the parameter in the extended phase. Due to duality these figures also show the spectrum in the localized phase with the interchange of $E_{r}$ and $E_{i}$. At the onset of localization, the spectrum with $E_{r}=E_{i}$ resembles that of the Harper equation. It is intriguing that even in this strong dissipative limit, extended states with real spectrum have a finite measure. In this lattice model with competing length scales, it therefore appears that extended states are essential for obtaining real eigenenergies. This is to be compared to earlier formal results where PT symmetry was a key for obtaining real spectrum for complex potentials.

We next discuss the weak dissipation limit, $\lambda_{r}=\lambda_{i} \equiv$ $\lambda$, described by the following tbm,

$$
\psi_{n+1}+\psi_{n-1}+2 \lambda e^{i \theta_{n}} \psi_{n}=E \psi_{n}
$$

As shown in figure (1), the system exhibits localization transition at $\lambda=.5$. The localization threshold is half of that of the Harper equation because both the real and the the imaginary part of the potential contributes towards localization. In the extended phase, the spectrum is found to be real and identical to that of $\lambda=0$ limit, namely $E=2 \cos \left(\theta_{n}\right)$. This explains the constant value of $\Delta E_{r}$ in the extended phase as seen in figure (1). In the localized phase, the eigenenergies are complex and appear to be described by the following expression,

$$
\begin{aligned}
& E_{r}=2 \cosh (\gamma) \cos \left(\theta_{n}\right) \\
& E_{i}=2 \sinh (\gamma) \sin \left(\theta_{n}\right)
\end{aligned}
$$

Here $\gamma$ is the inverse localization length of the system which is found to be equal to the corresponding value for the Harper equation $\gamma=\log (2 \lambda)$. In the limit $\lambda \rightarrow$ $\infty$, eigenenergies lie on a circle. Therefore, the localized phase is metastable with the lifetime determined by the localization length.

The FT of the model (5) can be analysed further as the transformation (2) reduces tridiagonal matrix to the following triangular matrix:

$$
2 \cos \left(\theta_{m}\right) \phi_{m}+2 \lambda \phi_{m-1}=E \phi_{m}
$$

With PBC, the eigenvalues of this triangular marix are the solution of the following algebraic equation:

$$
(2 \lambda)^{N}=\prod\left(E-2 \cos \left(\theta_{n}\right)\right)
$$

For $\lambda<0.5$, as $N \rightarrow \infty$, we obtain $E=2 \cos \left(\theta_{n}\right)$, which is found to be identical with numerically obtained spectrum of the model(5). For $\lambda>.5$, the FT of the model allows real solutions: $E=2 \cos \left(\theta_{n}\right)+2 \lambda$. These real energies were not found to be the solutions of the model (5). The FT of the model also exhibits complex solutions. It is easy to see that in $\lambda \rightarrow \infty$ limit, the algebric equation (9) has a solution where $E /(2 \lambda)$ lies on the unit circle which is also the solution for the model (5). This indicates a deep relationship between the spectra of the model (5) and its FT - the details have proven so far elusive.

Another aspect of model (5) is that at the onset to localization $\lambda=0.5$, the FT of the model describes the 
strong coupling limit of the fluctuations of the Harper equation once the exponentially decaying part is factored out [11]. This also describes the Ising model at the onset to long range order for $E=0$ [13. This limit has been shown to be universal using renormalization methods [1] as well as more rigorous analytic tools [12]. This result therefore establishes the multi-fractal character of the FT of the wave function at the onset of the localization transition. It should be noted that the eigenspectrum remains continuous at the localization transition in contrast with the Harper equation which is characterized by singular-continuous spectrum at the transition. Therefore, the $\lambda_{r}=\lambda_{i}$ limit of the model (1) provides a new class of incommensurate systems where the eigenspectrum is gapless and remains continuous except in the localized phase.

In summary, the localization transition of the Harper equation remains unaffected by the non-Hermitian perturbation. Weakly dissipative system are characterized by real eigenenergies in the extended phase. As the strength of the non-Hermitian potential increases, the number of extended states with real eigenenergies decrease approaching zero at the onset to localization. In the localized phase, states with complex spectrum have full measure. The localized phase is metastable, with state lifetimes determined by the localization length. The question of real eigenvalues is determined by both the transport character of the states as well as the amount of dissipation. An interesting result is that the extended states with real eigenenergies always have a positive measure while localized states with real energies have zero measure. Therefore, the measure of real eigenenergies provides a new order parameter for localizationdelocalization transition.

In contrast to the earlier results on localization in nonHermitian systems [4], the system studied here associates a complex spectrum with the localized phase. Further, the non-Hermitian potential does not delocalize the localized states.The localized phase of the Harper equation remains localized for any amount of non-Hermitian perturbation. This may be related with the fact that the non-Hermiticity appears in the diagonal part of the potential while the the non-Hermitian vector potential studied earlier [4 affects the off-diagonal part of the lattice model.

One future aspect of study is the extension of these results to a classical non-integrable (perhaps a kicked) system, thus investigating the effect of dissipation on non-integrable systems exhibiting localization. Furthermore, it should be noted that the equation (1) is the fermion representation of isotropic XY spin- $1 / 2$ chain in a complex magnetic field which is spatially modulating [13]. The consequences of the localization transition with complex spectrum on the magnetic properties of the system is another interesting open question. Finally, localization transition in incommensurate tightbinding lattice models corresponds to a transition to strange nonchatoic attractors (SNA) 14] in quasiperiodically driven maps. Therefore, the results of this paper may have important implications in the study of SNA's in complex maps.

The research of IIS is supported by a grant from National Science Foundation DMR 093296. We would like to thank Arjendu Pattanayak for useful discussions.

[1] e-mail: isatija@gmu.edu.

[2] P. G. Harper, Proc. Phys. Soc. London A 68, 874 (1955). For a review, see J. B. Sokoloff, Phys. Rep. 126, 189 (1985).

[3] C. Bender and S. Boettcher, Phys Rev Lett, 80 ( 1998)5243.

[4] N. Hatano and D. R Nelson, Phys. Rev. Lett. 77, 570 (1996); Phys Rev B 56, 8651 (1997).

[5] P.G Silvestrov, Report no. cond-mat/9804093.

[6] M. V Berry, J. Phys A: Math. Gen. 31(1998) 3493; M. Berry and O. Dell J. Phys. A, 31 (1998) 2093.

[7] K. Keller, M K Obertaler, S. Bernet, J Schmiedmayer and A Zeilinger, Phys Rev Lett, 79 (1997) 3327; Phys Rev Lett 77 (1996)4980.

[8] K. Schaadt and A. Kudrolli, Phys. Rev. E 60, 7203 (1999).

[9] D. Ferry and J. R. Barker, Applied Phys Lett, 74, 582 (1999).

[10] S. Aubry and G. André, Ann. Israel Phys. Soc. 3, 133 (1980).

[11] J. Ketoja and I. Satija, Phys Rev Lett, 75, 2712 (1995)

[12] " Golden mean renormalization for the Harper equation: the strong coupling fixed point" Ben Mestel, Andy Osbaldestin and B. Winn, preprint (2000).

[13] E. Lieb, T. Schultz, and D. Mattis, Ann Phys. (N.Y) 16, 407 (1961); I. I. Satija, Phys. Rev. B 48, 3511 (1993); Phys. Rev. B 49, 3391 (1994).

[14] A. Bondeson, E. Ott and T. M. Antonsen, Phys Rev Lett, 55 (1985) 2103; J. Ketoja and I. Satija Physica D, 1761, 1-11, (1997).

FIG. 1. (a) and (b) respectively show $\Delta E_{r}$ and $\Delta E_{i}$ vs $\lambda \equiv \lambda_{r}=\lambda_{i}$ for $\sigma=377 / 610$. The $\Delta E_{i}$ appears to be related to the derivative of $\Delta E_{r}$.

FIG. 2. (a) and (b) respectively show the variation in eigenvalues as a function of $\lambda \equiv \lambda_{r}=\lambda_{i}$ for $\mathrm{PBC}$ for $\sigma=34 / 55$. The extended phase of this non-Hermitian system exhibits real spectrum. Note that in contrast to the Harper equation, there is a bending and merging of levels at the transition. Furthermore, unlike Harper equation, the spectrum is not symmetric about $\lambda=0$. 
FIG. 3. (a) and (b) respectively show the variation in eigenvalues as a function of $\lambda \equiv \lambda_{r}$ for fixed $\lambda_{i}=.25$ for PBC for $\sigma=34 / 55$. In the extended phase all states have real eigenenergies provided $\lambda_{r} \geq \lambda_{i}$.

FIG. 4. Same as figure 2 and 3 for pure imaginary potential $\lambda_{r}=0$.

FIG. 5. shows the fraction of states with real eigenenergies as a function of $\lambda$ for $\lambda_{i}=.25$ case (a) and $\lambda_{r}=0$ (b). Here $\sigma=233 / 377$. The steps seen in this plot are due to finite size effects

FIG. 6. Figure shows the spectrum for differrent values of $\lambda$ for $\sigma=55 / 89$. For pure imaginary potential. Three verical columns respectively correspond to $\lambda=.5, .75,1$ : The three rows show (a) $E_{r}$ vs $E_{i}$ (b) $E_{r}$ vs n (c) $E_{i}$ vs n(sorted independently of the real part) 

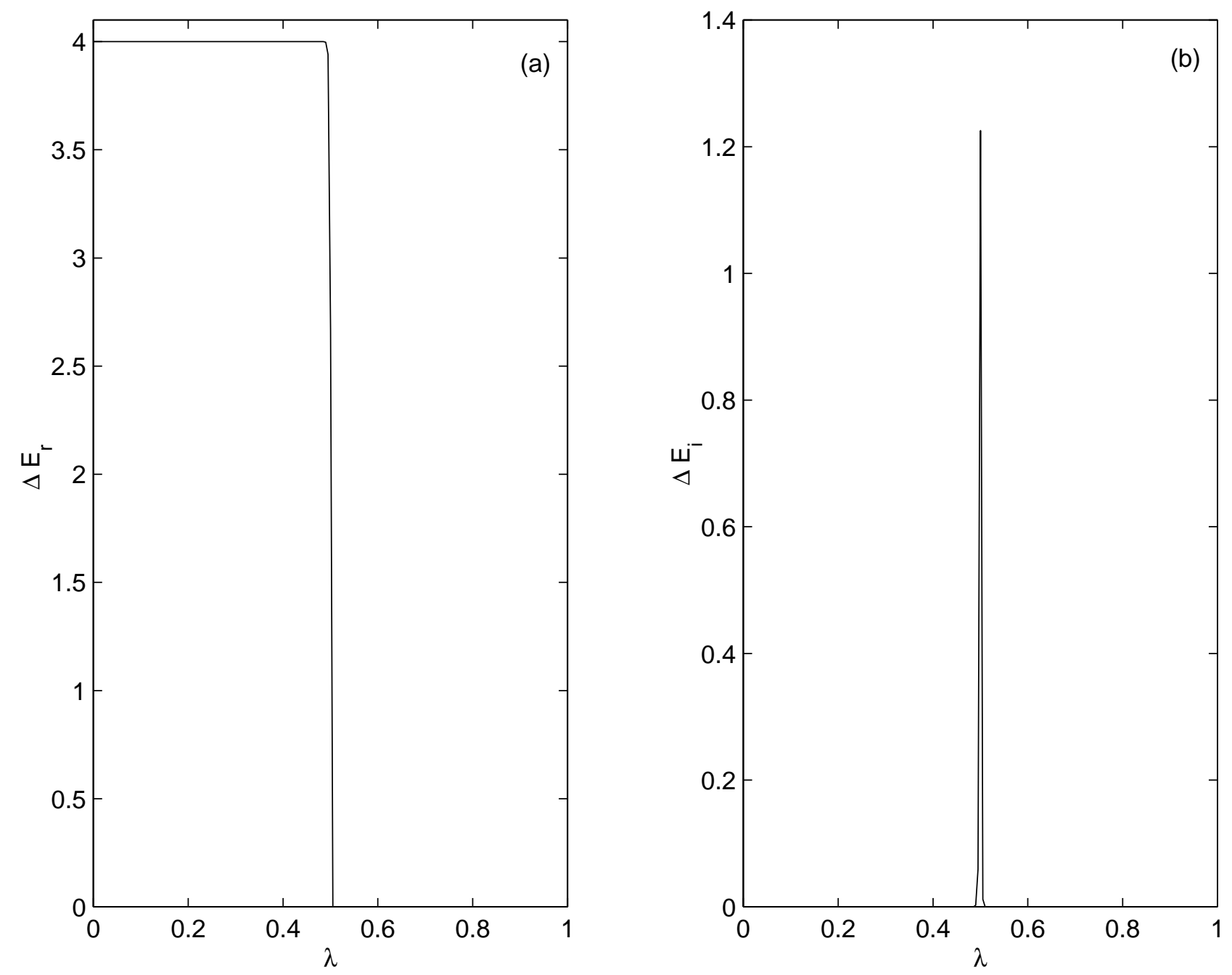

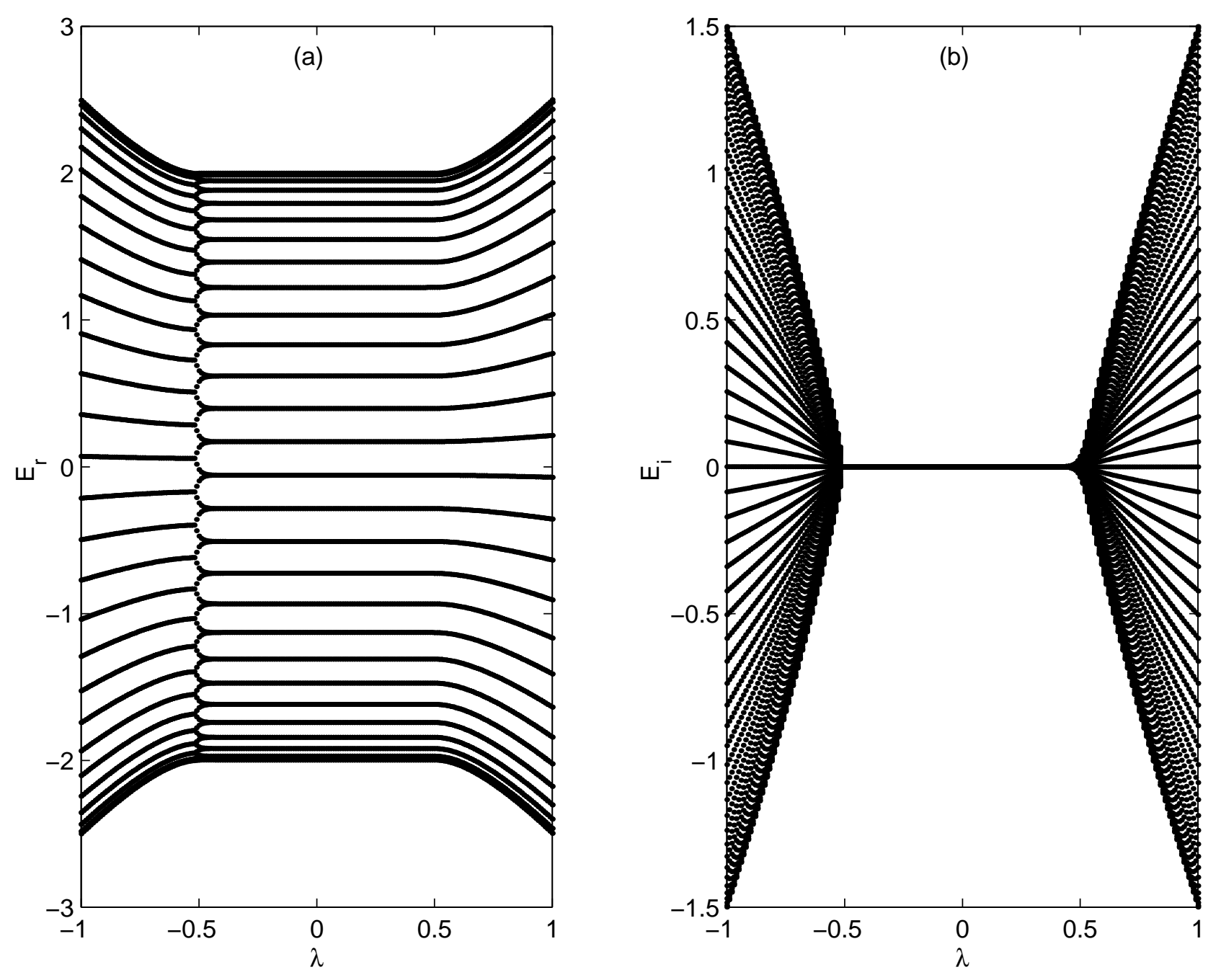

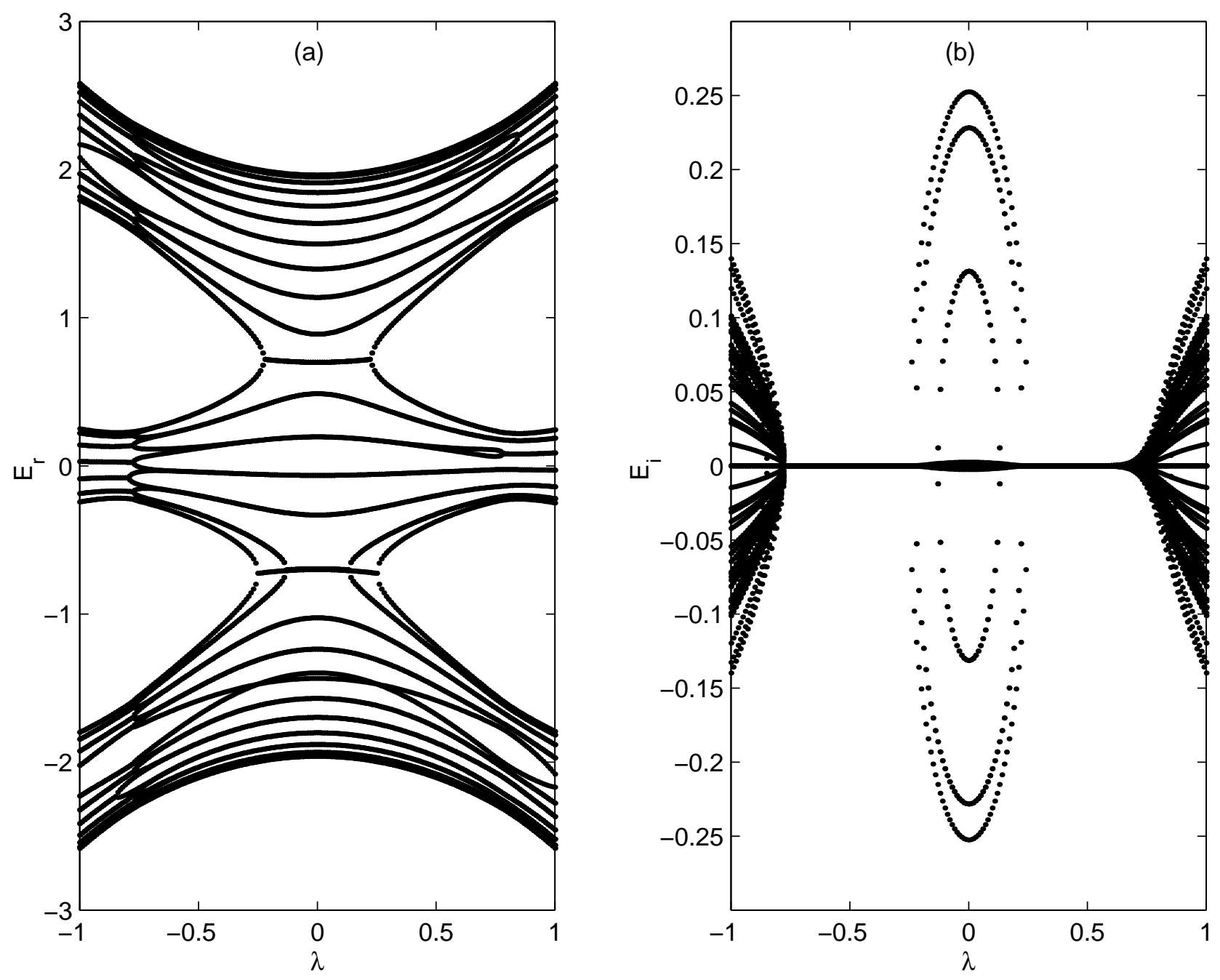

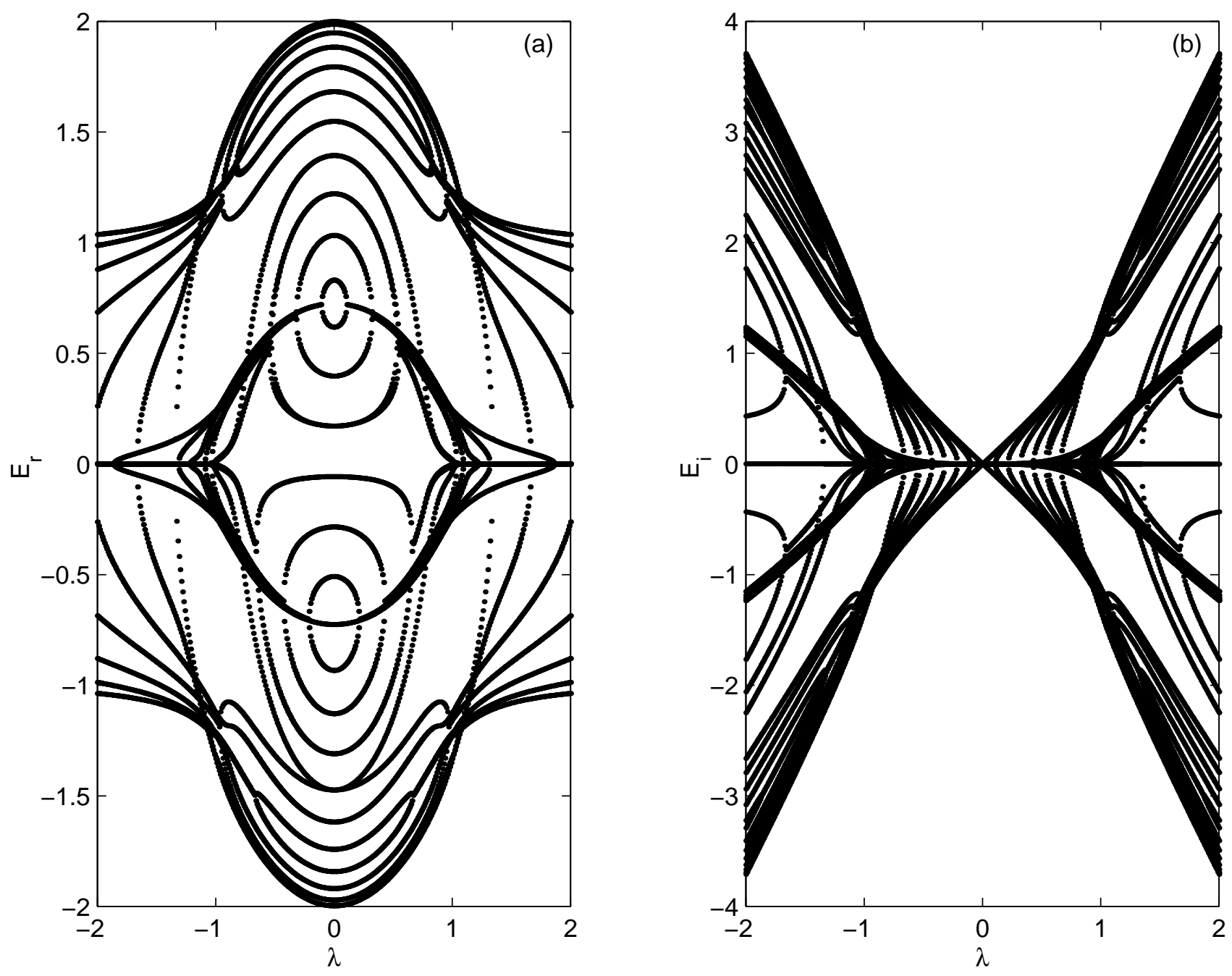

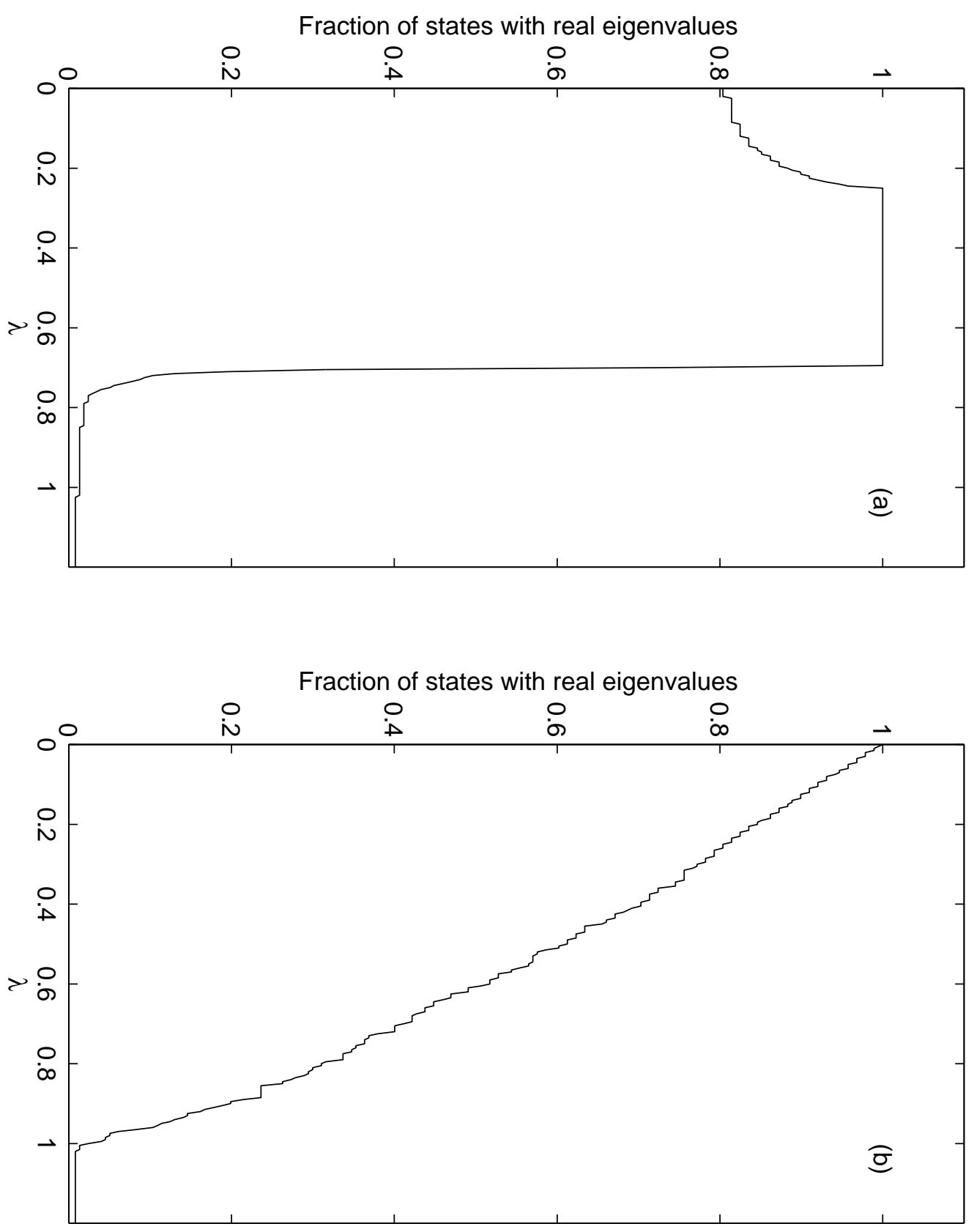

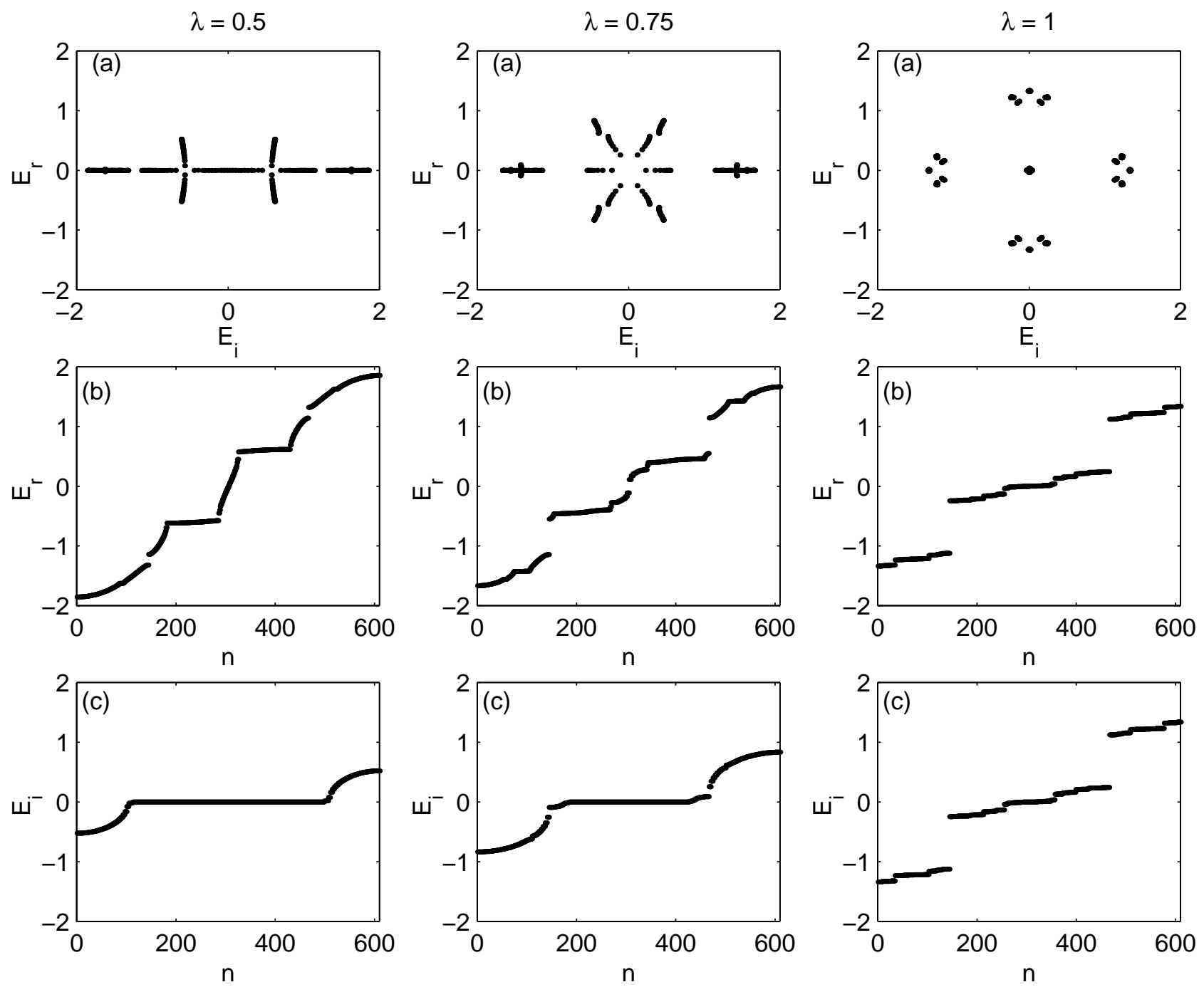